\title{
Rituximab in severe autoimmune TTP
}

A study conducted by the French

Thrombotic Microangiopathies Reference Center reports that rituximab may be safe and effective in patients with idiopathic thrombotic thrombocytopenic purpura (TTP) who have responded poorly to daily therapeutic plasma exchange (TPE). "Our work was motivated by the discovery that acquired TTP resulted from a severe, autoantibody-mediated, ADAMTS13 deficiency, as well as from previous preliminary works in which rituximab showed promising results," says Paul Coppo, lead investigator on the study.

The researchers enrolled 22 adults with TTP who had either refractory disease $(n=6)$ or disease exacerbation $(n=16)$ following standard TPE-based treatment. Patients were given add-on rituximab therapy—four infusions over 7 days. One patient with refractory disease died after two infusions of rituximab; the other 21 patients all received four rituximab infusions as first-line salvage therapy (a mean of 8.4 days after TPE initation) and achieved a durable remission. Time to durable remission was shorter in rituximab-treated patients than in 53 historical controls who had received TPE alone or with vincristine and survived (four other controls died). After 35 days, recovery of platelet count had occurred in all 21 rituximab-treated survivors but only $78 \%$ of control survivors. No relapses were recorded in the first year in the 19 rituximab-treated patients for whom data were available whereas $9.4 \%$ of controls relapsed during this time. Long-term (>1-year) relapse rates did not differ between groups. "Rituximab apparently decreases 1-year relapse rates by decreasing production of anti-ADAMTS13 antibodies," says Coppo. "However, longterm relapses may not be affected as the antibodies reappear."

Rebecca Ireland

Original article Froissart, A. et al. Efficacy and safety of first-line rituximab in severe, acquired thrombotic thrombocytopenic purpura with a suboptimal response to plasma exchange. Experience of the French Thrombotic Microangiopathies Reference Center. Crit. Care Med. 40, 104-111 (2012) 